\title{
Psychological interventions for posttraumatic stress disorder involving primary care physicians: systematic review and Meta-analysis of randomized controlled trials
}

Rebekka Gehringer ${ }^{1 * \dagger}$ (D), Antje Freytag ${ }^{1 \dagger}$, Markus Krause $^{1}$, Peter Schlattmann ${ }^{2}$, Konrad Schmidt $^{1}$, Sven Schulz ${ }^{1}$, Sophie Jana Zezulka ${ }^{1}$, Florian Wolf ${ }^{1}$, Jonas Grininger ${ }^{3}$, Mathias Berger ${ }^{4}$, Horst Christian Vollmar ${ }^{1,5+}$ and Jochen Gensichen ${ }^{3+}$

\begin{abstract}
Background: Evidence-based psychological interventions for posttraumatic stress disorder (PTSD) are available in specialized settings, but adequate care in primary care is often lacking.

The aim of this systematic review was to determine the effectiveness of psychological interventions for PTSD involving primary care physicians (PCPs) and to characterize these interventions as well as their providers.

Method: A systematic review and meta-analyses of randomized controlled trials (RCTs). Primary outcome were symptoms of PTSD.

Results: Four RCTs with a total of 774 patients suffering from PTSD symptoms were included, all applying cognitive behavioural based interventions. Three studies with psychological interventions being conducted by case managers were pooled in a meta-analysis. Interventions were not effective in the short term (0-6 months; SMD, $-0.1 ; 95 \% \mathrm{Cl}$, $\left.-0.24-0.04 ; 1^{2}=0 \%\right)$. Only two studies contributed to the meta-analysis for long term (12-18 months) outcomes yielding a small effect (SMD, $\left.-0.23 ; 95 \% \mathrm{Cl},-0.38--0.08 ;\left.\right|^{2}=0 \%\right)$.

Conclusions: Psychological interventions for PTSD in primary care settings may be effective in the long term but number and quality of included studies was limited so the results should be interpreted with caution.
\end{abstract}

Keywords: PTSD, Primary care, Systematic review

\footnotetext{
* Correspondence: rebekka.gehringer@med.uni-jena.de

${ }^{\dagger}$ Rebekka Gehringer, Antje Freytag, Horst Christian Vollmar and Jochen Gensichen contributed equally to this work.

${ }^{1}$ Institute of General Practice and Family Medicine, Jena University Hospital,

Friedrich-Schiller-University, Bachstr. 18, 07743 Jena, Germany

Full list of author information is available at the end of the article
}

(c) The Author(s). 2020 Open Access This article is licensed under a Creative Commons Attribution 4.0 International License, which permits use, sharing, adaptation, distribution and reproduction in any medium or format, as long as you give appropriate credit to the original author(s) and the source, provide a link to the Creative Commons licence, and indicate if changes were made. The images or other third party material in this article are included in the article's Creative Commons licence, unless indicated otherwise in a credit line to the material. If material is not included in the article's Creative Commons licence and your intended use is not permitted by statutory regulation or exceeds the permitted use, you will need to obtain permission directly from the copyright holder. To view a copy of this licence, visit http://creativecommons.org/licenses/by/4.0/ The Creative Commons Public Domain Dedication waiver (http://creativecommons.org/publicdomain/zero/1.0/) applies to the data made available in this article, unless otherwise stated in a credit line to the data. 


\section{Background}

Traumatic experiences can have long lasting effects on an individual's mental and physical well-being including the developments of PTSD. There is an ongoing discussion about the characteristics of this disease [1], being reflected by the changes of its diagnostic criteria in the new diagnostic systems DSM-5 (Diagnostic and Statistical Manual of Mental Disorders 5th edition) and ICD11 (International Statistical Classification of Diseases and Related Health Problems) [2, 3]. While ICD-11 requires three key symptom clusters of re-experiencing, avoidance and hyperarousal, DSM-5 added a fourth symptom cluster of persistent negative alterations in cognitions and mood.

PTSD lifetime prevalence is estimated between 1.3$8.8 \%$ in the World Mental Health Surveys [4]. The three most common causes of PTSD in the World Mental Health Surveys were rape, other sexual assault and unexpected death of a loved one [5].

Several evidence-based therapies for the treatment of PTSD are available to patients: cognitive- behaviouralbased therapies (i.e. exposure therapies), eye movement desensitization and reprocessing (EMDR), and narrative exposure therapy (NET) [6]. Evidence suggests that trauma-focused therapies (i.e. exposure therapies, cognitive processing therapy, EMDR) may be more effective than non-trauma-focussed therapies [6, 7]. Pharmacological treatment using selective serotonin reuptake inhibitors may be helpful when trauma focused psychotherapies are not available or are refused by the patient [8]. Irrespective of the cause of trauma, most patients initially turn to general practitioners/primary care physicians (PCP - for reasons of consistency we refer to PCP in the further text) [9], with a median point prevalence of PTSD in primary care patients of $12.5 \%$ [10].

Despite the existence of effective interventions, there is a relevant time lag until patients receive specialist care. In the United States the median period of delay is 12 years between the onset of PTSD and first treatment contact (defined as talking about the disorder to any professional) [11]. Reasons for the delay in accessing treatment include the stigma associated with mental health care as well as cultural and institutional attitudes [12]. Additionally, there are structural impediments such as shortages of psychotherapists and inefficient allocation of patients to providers. To improve recognition and treatment of PTSD, several new approaches have been developed (e.g. internet based therapies, self-help, collaborative care) $[13,14]$.

National guidelines highlight that patients suffering from PTSD initially contact PCPs, who are in charge of diagnosing PTSD and organising care $[15,16]$. There are several advantages in primary care settings which may facilitate the initiation and reduce barriers to psychological therapies: Treatment can be provided lowthreshold and early starting compared to secondary care settings. Many patients have established a trustful relationship with their PCPs, thus an important first step within psychotherapeutic work has already been achieved [17]. PCP's knowledge of their patients' personal environment and resources can be an important resource during therapy $[18,19]$. In addition, PCPs may help to close the large gap between supply and demand for the support of traumatized people [20].

Several other mental health disorders have effectively been treated in primary care, including depression and anxiety [21]. Important components for successful treatment include case management delivered by case managers (CM) with mental health training, scheduled supervision of CMs by mental health specialists, and the coordinated involvement of PCPs, CMs, and mental health specialists [22-24].

Two narrative reviews described the rationale for the management of PTSD in a primary care setting involving brief cognitive behavioural therapies (CBT); utilizing self-help and internet based approaches; as well as collaborative care $[13,14]$. Based on a narrative review, Hoeft et al. suggest that collaborative care that offers psychotherapy is a promising approach [25]. The three reviews give an overview on diverse interventions investigated in a wide range of settings and with multiple designs. Given the heterogeneity of interventions, a quantitative analysis was not performed. A meta-analysis is needed to increase evidence on psychological treatment for PTSD in a primary care setting and to investigate specific intervention effects.

\section{Objectives}

The aim of this review was to determine the effectiveness of psychological interventions for PTSD involving PCPs. The second aim was to characterize these interventions and their providers, and to describe the providers' specific tasks, as well as their interaction.

\section{Methods}

Objectives, inclusion criteria, and methods were prespecified in a study protocol registered with Prospero (Registration number CRD42017060123).

\section{Eligibility criteria}

Both cluster and individually RCTs were included applying psychological interventions to reduce PTSD symptoms. Participants aged 18 and older with a PTSD diagnosis (according to a valid diagnostic system, e.g. DSM-4 or 5, ICD-10) or with clinically relevant PTSD symptoms, determined by validated instruments, were considered. 
Eligible interventions were: CBTs (cognitive therapy, cognitive processing therapy, cognitive restructuring therapy, coping skills training, exposure therapy including prolonged exposure and dialectical behavioural therapy), EMDR, NET and others such as writing therapy, hypnotherapy, interpersonal therapy, present centred therapy, eclectic psychotherapy and psychodynamic therapies [6, 26-30]. Application of case management only or other service delivery models such as collaborative care without the implementation of one of the above listed psychological interventions was excluded. To be eligible for inclusion, psychological therapies had to be delivered by PCPs, or by non-physician primary care providers, on condition that PCPs remained actively engaged in the intervention. Active engagement could mean treatment was linked to the monitoring, the clinical instructions, the supervision, or the advice of the PCP, or the therapist and the physician used a shared patient chart, or the PCP regularly received feedback on the therapy's progress and made recommendations for further management. Control groups may have also received active interventions such as case management, training of PCPs and collaborative care approaches without the implementation of the above listed psychological interventions.

To be eligible the primary outcome had to measure PTSD symptoms using validated instruments.

We did not apply any restrictions concerning language, publication status, or year of publication.

\section{Search methods}

The full electronic search strategy for Medline is published in additional file 1 . To identify studies we searched electronic databases (Medline, Embase, PsycINFO, the Cochrane Central Register of Controlled trials and CINAHL) from their inception until November 2016. An update search was performed for the period from December 2016 to February 2019. Additionally, we screened several conference proceedings where content was available online (see additional file 1). We also searched the International Clinical Trials Registry Platform and reference lists from included studies and relevant reviews.

\section{Study selection and data extraction}

Eligibility assessment was performed independently from RG and MK/SJZ. After screening abstract and title, 245 full texts were reviewed.

The data extraction form was developed on the basis of the EPOC data collection form and checklist, and the EQUATOR template for intervention description and replication (TIDieR) checklist $[31,32]$. Due to the limited number of included studies the extraction form was piloted with one study. We received data from the main study and, if available, study design and protocols, as well as further publications reporting relevant outcomes. Two reviewers conducted outcome extraction independently (RG/JG). The primary outcome assessed was PTSD symptoms. Secondary outcomes recorded were comorbidities, quality of life, psychopharmacologic medication use, mental health care use, adverse events, patients` satisfaction, additional costs for intervention, treatment and medication adherence, and suicidality. The remaining data (i.e. quality criteria, components of the Chronic Care Model [33]) were extracted by RG and checked by MK. Additionally, we focused on the involvement of PCPs and extracted their profession, PTSD specific training, tasks performed, and their interaction with other providers. All disagreements occurring during study selection and data extraction were resolved through discussion. If no agreement could be reached, a team of authors made the decision (AF, RG, JG1, MK, $\mathrm{SvS}, \mathrm{KS}, \mathrm{HCV})$. When any information was missing the corresponding authors were contacted. 12 of the 22 contacted authors responded, but could not provide additional quantitative outcome data.

\section{Risk of bias assessment}

Risk of bias of included studies was assessed by two independent reviewers (RG/MK) using the Cochrane Risk of Bias Tool [34]. Resulting disagreements were resolved through discussion and with a third reviewer (AF).

The quality of evidence was assessed using GRADE [35].

\section{Statistical analysis}

We estimated standardized mean differences with Cohen's $d$ due to different scales for PTSD symptoms among studies. Heterogeneity was quantified using $\mathrm{I}^{2}$ statistics and linear mixed models. Quantitative analyses were performed using $\mathrm{R}$ and SAS 9.4. Due to the absence of statistical heterogeneity, we used the fixed effects model for performing the meta-analysis. Because standard deviations for means of the primary outcome were neither reported nor could be calculated from the presented data in one study [36], and could not be provided by the authors, we used the reported standard deviations for baseline means also for the follow-up data. To additionally assess the effects of time, intervention, and time-intervention interaction on the primary outcome we applied a linear mixed model using a restricted maximum likelihood method on a Gaussian distribution. The model selection was based on the Bayesian information criterion (BIC). We chose the model with the lowest BIC. All calculations for regression analyses were performed using SAS (proc GLIMMIX) procedure.

Other than announced in the protocol, no further analyses (meta-regression, sensitivity analyses) were 
conducted because of the limited number of included studies. Publication bias was estimated considering the inclusion of small and negative effect studies. A funnel plot could not be developed because of the small number of studies included.

\section{Results}

\section{Study selection and study characteristics}

We identified 5996 records in electronic databases during our search (flowchart additional file 2). An additional 48 records were found through searches in reference lists, conference proceedings, and the International Clinical Trials Registry Platform. After removing duplicates we screened 4418 records. Two hundred fourty-five fulltexts were assessed for eligibility. Finally, 4 RCTs (STEP S-UP: Stepped Enhancement of PTSD Services Using Primary Care [36], DESTRESS-PC: Delivery of Self Training and Education for Stressful Situations-Primary Care version [37], CALM: coordinated anxiety learning and management [38, 39], PE-PC: Prolonged Exposure for Primary Care [40]) were included in this review with 774 participants suffering from PTSD. All studies were multicentre trials.

We extracted data from 16 reports (including research protocols, study designs and analysis of secondary data).
Study characteristics (Table 1): STEPS-UP [36] was performed on active-duty military members with $80.9 \%$ male participants in US-military primary care clinics. Relevant PTSD symptoms were measured with the PCLC (PTSD Checklist-Civilian version) for inclusion. Details of further inclusion and exclusion criteria of single studies are summarised in additional file 3 . This was the only study reporting adverse effects and no case of adverse effects was noted [36]. The intervention was a stepped-care model based on CBT, using nurse-assisted, online or telephone self-management in Step 2, and the possibility of mental health specialists delivered psychotherapy in the last step (Table 2). Psychological therapy lasted 6-9 weeks. Applied strategies to improve treatment adherence were motivational interviewing (MI) and behavioural activation (BA). The control group received collaborative care as usual care, which had been implemented within the military health care system previously and consisted of prepared primary care practices, care management and enhanced mental health specialty. PCPs prescribed psychoactive medications in both groups.

DESTRESS-PC [37] participants were recently deployed military service members and veterans. Most participants were male $(81.3 \%)$. The study was set in

Table 1 Study characteristics

\begin{tabular}{|c|c|c|c|c|}
\hline Study & STEPS-UP & DESTRESS-PC & CALM & PE-PC \\
\hline Country & US & US & US & US \\
\hline Setting & 18 Army primary care clinics & $\begin{array}{l}\text { Dep. Defense and Veterans } \\
\text { Affairs primary care clinics }\end{array}$ & 17 primary care clinics & $\begin{array}{l}\text { two military } \\
\text { treatment facilities }\end{array}$ \\
\hline Population & $\begin{array}{l}\text { active duty US military members } \\
\text { with PTSD and/or depression }\end{array}$ & $\begin{array}{l}\text { recently deployed military } \\
\text { service members/ veterans } \\
\text { with PTSD }\end{array}$ & $\begin{array}{l}\text { primary care patients with } \\
P D, G A D, S A D, P T S D \text { or all } 4\end{array}$ & $\begin{array}{l}\text { active duty military } \\
\text { service members with } \\
\text { (subthreshold) PTSD }\end{array}$ \\
\hline Male gender No. (\%) & $539(80.9 \%)$ male $^{a}$ & $65(81.3 \%)$ male & $290(28.9 \%)$ male $^{b}$ & $50(75 \%)$ male \\
\hline Age in years & $31.2^{\mathrm{a}}$ & 36.5 & $43.5^{b}$ & 40 \\
\hline Sample size IG/CG No. & $285 / 281^{c}$ & $43 / 37$ & $33 / 28^{d}$ & $34 / 33$ \\
\hline Psychological intervention & $\begin{array}{l}\text { Online/ telephone delivered } \\
\text { CBT-based therapies; other } \\
\text { evidence based psychotherapies }\end{array}$ & $\begin{array}{l}\text { CBT based nurse-assisted, } \\
\text { online self-management } \\
\text { tool }\end{array}$ & $\begin{array}{l}\text { computerized CBT program } \\
\text { tailored to the } 4 \text { specific } \\
\text { anxiety disorders }\end{array}$ & $\begin{array}{l}\text { brief Prolonged } \\
\text { Exposure for } \\
\text { Primary Care }\end{array}$ \\
\hline Involved providers & CM, PCP, MHS & CM, PCP, MHS & CM, PCP, MHS & PCP, MHS \\
\hline PTSD diagnosis instrument & $\begin{array}{l}\text { PTSD Checklist- Civilian version } \\
\text { (PCL-C) }\end{array}$ & $\begin{array}{l}\text { Clinician administered } \\
\text { PTSD scale (CAPS) }\end{array}$ & $\begin{array}{l}\text { Mini International } \\
\text { Neuropsychiatric Interview }\end{array}$ & $\begin{array}{l}\text { PTSD Checklist- } \\
\text { Stressor Specific } \\
\text { version (PCL-S) }\end{array}$ \\
\hline $\begin{array}{l}\text { PCL-C at baseline, IG/CG, } \\
\text { mean (SD) }\end{array}$ & $58.5(11.1) / 57.7(10.8)^{a}$ & $55.16(10.89) / 58.56(10.01)$ & $57.15(12.56)^{d} / 56.90(12.57)^{d}$ & $\begin{array}{l}49,8(12,8) / 52,2 \\
(14,1) \mathrm{e}\end{array}$ \\
\hline $\begin{array}{l}\text { Psychiatric comorbidity } \\
\text { outcome measures }\end{array}$ & $\begin{array}{l}\text { SCL-20 (depression), AUDIT } \\
\text { (alcohol consumption), PHQ-15 } \\
\text { (somatic disorder), BPI (pain) }\end{array}$ & $\begin{array}{l}\text { PHQ-8 (depression), } \\
\text { PHQ-15 (somatic disorder) }\end{array}$ & $\begin{array}{l}\text { GADSS (GAD), PDSS-SR (PD), } \\
\text { SPIN (SAD), PHQ-8 (depression), } \\
\text { BSI (somatization and anxiety) }\end{array}$ & PHQ-9, BHM \\
\hline
\end{tabular}

$C M$ care manager, $P C P$ primary care physician, $M H S$ mental health specialist, $C B T$ cognitive behavioural therapy, $P D$ panic disorder, GAD generalized anxiety disorder, $S A D$ social anxiety disorder;

a patients with PTSD and/or depression;

b all anxiety disorders (PD, GAD, SAD, PTSD);

c patients with PTSD;

d patients who selected PTSD as their principal disorder

e PTSD Checklist-Stressor Specific version (PCL-S) 
Table 2 Characteristics of the intervention

\begin{tabular}{|c|c|c|c|c|c|c|c|}
\hline \multirow{2}{*}{$\begin{array}{l}\text { Study } \\
\text { IC/CG }\end{array}$} & & \multicolumn{4}{|l|}{ STEPS-UP } & \multicolumn{2}{|l|}{ DESTRESS-PC } \\
\hline & & IG & & & CG & IG & CG \\
\hline \multirow{8}{*}{$\begin{array}{l}\text { Psychological } \\
\text { intervention }\end{array}$} & Description of & STEP 3: & STEP 2: & STEP 1: & collaborative care & CBT-based \& & \\
\hline & & psychotherapy & $\begin{array}{l}\text { CBT based self- } \\
\text { management }\end{array}$ & $\begin{array}{l}\text { care } \\
\text { manage- } \\
\text { ment (edu- } \\
\text { cation, BA, } \\
\text { MI) }\end{array}$ & $\begin{array}{l}\text { Without implementation } \\
\text { of psychological } \\
\text { therapies }\end{array}$ & $\begin{array}{l}\text { Stress } \\
\text { inoculation } \\
\text { training in a } \\
\text { nurse-guided } \\
\text { online patient } \\
\text { self- } \\
\text { management } \\
\text { paradigm }\end{array}$ & $\begin{array}{l}\text { Intensity } \\
\text { CM and } \\
\text { training of } \\
\text { PCPs }\end{array}$ \\
\hline & $\begin{array}{l}\text { who received } \\
\text { the intervention }\end{array}$ & $\begin{array}{l}\text { patients' request, high } \\
\text { risk patients, } \\
\text { unresponsive to STEP } \\
1+2, \text { PCPs decision }\end{array}$ & $\begin{array}{l}\text { patients who } \\
\text { remain } \\
\text { clinically } \\
\text { symptomatic } \\
\text { after 3-6 weeks }\end{array}$ & all patients & all patients & all patients & all patients \\
\hline & $\begin{array}{l}\text { who delivered } \\
\text { the intervention }\end{array}$ & local MHS & $\mathrm{CM}$ & $C M, P C P$ & $C M, P C P, M H S$ & $\begin{array}{l}\text { CM/computer } \\
\text { program }\end{array}$ & CM/PCP \\
\hline & $\begin{array}{l}\text { method of } \\
\text { delivery }\end{array}$ & $\begin{array}{l}\text { in-person or via } \\
\text { telephone }\end{array}$ & $\begin{array}{l}\text { online or via } \\
\text { telephone }\end{array}$ & $\begin{array}{l}\text { via } \\
\text { telephone, } \\
\text { electronic } \\
\text { messaging, } \\
\text { in-person }\end{array}$ & via telephone, & $\begin{array}{l}\text { online, via } \\
\text { telephone, E- } \\
\text { Mail, in-person }\end{array}$ & $\begin{array}{l}\text { via } \\
\text { telephone, } \\
\text { E-Mail, in- } \\
\text { person }\end{array}$ \\
\hline & $\begin{array}{l}\text { duration of the } \\
\text { intervention }\end{array}$ & not reported & 6-9 weeks & 12 months & 12 months & $\begin{array}{l}\text { 6-max. } 10 \\
\text { weeks }\end{array}$ & $\begin{array}{l}\text { not } \\
\text { reported }\end{array}$ \\
\hline & $\begin{array}{l}\text { number of } \\
\text { contacts }\end{array}$ & not reported & $3-9$ & $\min .12$ & $\min .12$ & $\begin{array}{l}\text { log in } 3 \text { times } \\
\text { /week, number } \\
\text { of CM-contacts } \\
\text { not reported }\end{array}$ & $\begin{array}{l}3 \\
\text { telephone } \\
\text { check-ins, } \\
\text { risk assess- } \\
\text { ment at } \\
\text { weeks 2/4/ } \\
6\end{array}$ \\
\hline & $\begin{array}{l}\text { strategies } \\
\text { applied to } \\
\text { sustain/ improve } \\
\text { treatment } \\
\text { adherence }\end{array}$ & \multicolumn{3}{|c|}{$\mathrm{CM}$ were trained in $\mathrm{BA}$, problem solving and $\mathrm{Ml}$} & $\begin{array}{l}\text { Adherence was } \\
\text { monitored }\end{array}$ & not reported & $\begin{array}{l}\text { not } \\
\text { reported }\end{array}$ \\
\hline \multirow[t]{2}{*}{$\begin{array}{l}\text { Pharmacological } \\
\text { intervention }\end{array}$} & $\begin{array}{l}\text { interventions for } \\
\text { improved } \\
\text { pharmacological } \\
\text { treatment }\end{array}$ & see STEP 2 & $\begin{array}{l}\text { Expert training } \\
\text { in } \\
\text { pharmacologic } \\
\text { treatment for } \\
\text { PCPs }\end{array}$ & $\begin{array}{l}\text { not } \\
\text { reported }\end{array}$ & $\begin{array}{l}\text { Stepped } \\
\text { pharmacological } \\
\text { treatment }\end{array}$ & no intervention & $\begin{array}{l}\text { no } \\
\text { intervention }\end{array}$ \\
\hline & $\begin{array}{l}\text { who prescribed } \\
\text { medication }\end{array}$ & PCP & & & PCP & not reported & $\begin{array}{l}\text { not } \\
\text { reported }\end{array}$ \\
\hline \multicolumn{2}{|l|}{ Study } & \multicolumn{3}{|l|}{ CALM } & PE-PC & & \\
\hline IC/CG & & IG & CG & & IG & \multicolumn{2}{|l|}{ CG } \\
\hline \multirow[t]{5}{*}{$\begin{array}{l}\text { Psychological } \\
\text { intervention }\end{array}$} & $\begin{array}{l}\text { Description of } \\
\text { the intervention }\end{array}$ & $\begin{array}{l}\text { computer-assisted } \\
\text { CBT program }\end{array}$ & \multicolumn{2}{|c|}{$\begin{array}{l}\text { usual care by } P C P \text {, referral to } \\
\text { MHS possible }\end{array}$} & $\begin{array}{l}\text { brief Prolonged Exposure } \\
\text { for Primary Care }\end{array}$ & \multicolumn{2}{|c|}{ minimal contact group } \\
\hline & $\begin{array}{l}\text { who received } \\
\text { the intervention }\end{array}$ & $\begin{array}{l}\text { patients could choose } \\
\text { computer-assisted } \\
\text { CBT medication, or } \\
\text { both }\end{array}$ & \multicolumn{2}{|l|}{ all patients } & all patients & \multicolumn{2}{|l|}{ all patients } \\
\hline & $\begin{array}{l}\text { who delivered } \\
\text { the intervention }\end{array}$ & CM (ACS) & \multicolumn{2}{|l|}{ PCP, MHS } & PCP, MHS & \multicolumn{2}{|l|}{ PCP, MHS } \\
\hline & $\begin{array}{l}\text { method of } \\
\text { delivery }\end{array}$ & $\begin{array}{l}\text { in-person (CBT), via } \\
\text { telephone (follow-up) }\end{array}$ & \multicolumn{2}{|c|}{ in-person, via telephone } & in-person & \multicolumn{2}{|l|}{ via telephone } \\
\hline & $\begin{array}{l}\text { duration of the } \\
\text { intervention }\end{array}$ & $\begin{array}{l}10 \text { to } 12 \text { weeks, } \\
\text { symptomatic } \\
\text { participants could }\end{array}$ & \multicolumn{2}{|l|}{ not reported } & $\begin{array}{l}30 \text { min appointments } \\
\text { delivered over 4-6 weeks }\end{array}$ & \multicolumn{2}{|l|}{6 weeks } \\
\hline
\end{tabular}


Table 2 Characteristics of the intervention (Continued)

\begin{tabular}{|c|c|c|c|c|c|}
\hline & & $\begin{array}{l}\text { receive up to } 3 \text { more } \\
\text { steps (i.e., another } \\
10-12 \text { weeks) of } \\
\text { treatment }\end{array}$ & & & \\
\hline & $\begin{array}{l}\text { number of } \\
\text { contacts }\end{array}$ & $\begin{array}{l}\text { CBT: } 6 \text { to } 8 \text { weekly } \\
\text { sessions }\end{array}$ & not reported & 4 & 6 \\
\hline & $\begin{array}{l}\text { strategies } \\
\text { applied to } \\
\text { sustain or } \\
\text { improve } \\
\text { treatment } \\
\text { adherence }\end{array}$ & $\begin{array}{l}\text { ACS received } \\
\text { didactics of } \mathrm{Ml}\end{array}$ & not reported & $\begin{array}{l}\text { review by an } \\
\text { independent clinician } \\
\text { using adherence rating } \\
\text { forms }\end{array}$ & not reported \\
\hline \multirow[t]{2}{*}{$\begin{array}{l}\text { Pharmacological } \\
\text { intervention }\end{array}$} & $\begin{array}{l}\text { interventions for } \\
\text { improved } \\
\text { pharmacological } \\
\text { treatment }\end{array}$ & $\begin{array}{l}\text { single-session } \\
\text { medication } \\
\text { management training } \\
\text { for PCPs using a } \\
\text { simple algorithm, } \\
\text { adherence } \\
\text { monitoring by ACS } \\
\text { for medication } \\
\text { management }\end{array}$ & not reported & $\begin{array}{l}\text { psychotropic medication } \\
\text { should remain } \\
\text { unchanged throughout } \\
\text { the intervention }\end{array}$ & $\begin{array}{l}\text { psychotropic medication } \\
\text { should remain unchanged } \\
\text { throughout the intervention }\end{array}$ \\
\hline & $\begin{array}{l}\text { who prescribed } \\
\text { medication }\end{array}$ & PCP & PCP & not reported & not reported \\
\hline
\end{tabular}

IG intervention group, $C G$ control group, $C M$ care manager, $P C P$ primary care physician, $M H S$ mental health specialist, $A C S$ anxiety clinical specialist, $C B T$ cognitive behavioural therapy, $B A$ behavioural activation, $M I$ motivational interviewing

Department of Defense and Veterans Affairs primary care clinics. The clinician administered PTSD scale (CAPS) was used for diagnosis. Similar to STEPS-UP, a CBT based, nurse-guided, online self-management paradigm constituted the intervention, which lasted 6-10 weeks. The control group received usual care which was optimized by training of PCPs in PTSD identification and treatment and basic care management including phone check-ins to monitor symptoms and feedback to providers.

The CALM trial $[38,39]$ was set in US-primary care clinics and included 28.9\% male participants. Diagnosis instrument was the Mini International Neuropsychiatric Interview. The intervention consisted of a computerassisted, face-to-face treatment for 10-12 weeks and could be extended up to 3 times. MI was used to increase treatment adherence. The control group received the usual PCP care with the option of mental health specialist referral.

PE-PC [40] was performed on active-duty military members with $75 \%$ male participants in two US military treatment facilities. Relevant PTSD symptoms were measured with the PCL-S (PTSD Checklist- Stressor Specific version) for inclusion. The intervention was based on a brief protocol for prolonged exposure developed for a primary care setting. Behavioural health consultants (BHCs) working in the primary care team were specially trained to deliver the intervention. Psychological therapy lasted 4-6weeks. The control group was contacted weekly by the BHCs to monitor their status and was offered to receive PE-PC also after 6 weeks. The BHCs worked as a consultant to the PCP.
Involved providers (Table 3): PCPs always received feedback on the ongoing therapy and special training, except for PE-PC (no PCP training). They were responsible for the pharmacological treatment and were supervised by mental health specialists in two studies [36, 38, 39]. CMs (registered nurses, nurses with a bachelor or a master of science in nursing, psychiatric mental health nurse practitioners, social workers, counsellors and psychologists) had a central role in three studies [36-39]. They delivered or assisted the psychological therapies, coordinated care and communication between PCPs and mental health specialists, educated patients, delivered MI, BA and counselling, and monitored symptoms. Mental health specialists supervised CMs in three studies and pharmacological treatment in two studies [36, 38, 39]. In STEPS-UP they developed recommendations together with the $\mathrm{CM}$ and delivered psychotherapies for patients in STEP 3. Only in PE-PC the intervention was delivered by specially trained BHCs and no case management was applied. Due to the very different therapy concepts, this study was not included in the meta-analysis.

\section{Results of individual studies}

The individual results of three RCTs included in the meta-analysis in the short (0-6 months) and long term ( $\geq 12$ months) are presented with the forest plot in Fig. 1.

\section{Synthesis of results}

In a meta-analysis we pooled the PTSD-symptom shortterm (0-6 months) outcomes from three studies. 
Table 3 Involvement of treatment providers in the intervention

\begin{tabular}{|c|c|c|c|c|c|}
\hline Study & $\begin{array}{l}\text { Treatment } \\
\text { providers }\end{array}$ & Profession & $\begin{array}{l}\text { Special training for } \\
\text { intervention }\end{array}$ & Tasks and interaction with other providers & $\begin{array}{l}\text { Supervision } \\
\text { received }\end{array}$ \\
\hline \multirow[t]{4}{*}{ STEPS-UP } & PCP & not reported & $\begin{array}{l}\text { Expert training in the } \\
\text { pharmacologic treatment of } \\
\text { depression and PTSD }\end{array}$ & $\begin{array}{l}\text { - provision of information related to } \\
\text { treatment options } \\
\text { - providing evidence based } \\
\text { pharmacotherapy } \\
\text { - selection of the next step for a patient's } \\
\text { treatment plan (with CM assistance)- } \\
\text { implementation of central teams' } \\
\text { recommendations- receives feedback from } \\
\text { CMs and the central team }\end{array}$ & $\%$ \\
\hline & CM & $\begin{array}{l}\mathrm{RN} ; \\
\text { social workers } \\
\text { counsellors }\end{array}$ & $\begin{array}{l}\text { trained and coached weekly } \\
\text { by telephone in BA, problem } \\
\text { solving, and Ml, training in } \\
\text { the web-based intervention }\end{array}$ & $\begin{array}{l}\text { - coordinating care between involved providers } \\
\text { - improving patients activation and } \\
\text { engagement in their care (education, } \\
\text { MI, BA) } \\
\text { - assistance of patients and PCPs in } \\
\text { choosing treatment options } \\
\text { - assistance with web-based or delivery of } \\
\text { telephone CBT self-management }\end{array}$ & by MHS \\
\hline & MHS & $\begin{array}{l}\text { psychiatrists; } \\
\text { psychologists; } \\
\text { clinical social workers }\end{array}$ & $\begin{array}{l}\text { trained in empirically } \\
\text { validated psychotherapies for } \\
\text { PTSD and depression }\end{array}$ & $\begin{array}{l}\text { - delivery of empirically validated } \\
\text { psychotherapy } \\
\text { - review of patients' medication } \\
\text { - providing CM caseload reviews } \\
\text { - training and supervision of CMs. }\end{array}$ & $\begin{array}{l}\text { by } \\
\text { psychotherapist }\end{array}$ \\
\hline & $\begin{array}{l}\text { central } \\
\text { team }\end{array}$ & $\begin{array}{l}\text { CM; psychiatrist; } \\
\text { psychologist; } \\
\text { administrative } \\
\text { support }\end{array}$ & not reported & $\begin{array}{l}\text { - coordination and supervision of the } \\
\text { intervention } \\
\text { - development of recommendations for PCPS } \\
\text { - reformulation of CM engagement strategies } \\
\text { - ensure appropriate medication }\end{array}$ & $\%$ \\
\hline \multirow[t]{3}{*}{ DESTRESS-PC } & PCP & not reported & $\begin{array}{l}\text { pre-study didactic training } \\
\text { regarding management of } \\
\text { and clinical tools for PTSD } \\
\text { and associated conditions }\end{array}$ & $\begin{array}{l}\text { - treatment of patients with feedback } \\
\text { from CM }\end{array}$ & $\%$ \\
\hline & $\begin{array}{l}C M / D E S T \\
\text { RESS nurse }\end{array}$ & $\begin{array}{l}\text { RN; MSN; BSN; } \\
\text { PMHNP-BC }\end{array}$ & not reported & $\begin{array}{l}\text { - assistance with the web-based DESTRESS-PC } \\
\text { interface } \\
\text { - monitoring of compliance and symptom } \\
\text { levels } \\
\text { - reengagement of participants with } \geq 2 \text { missed } \\
\text { logons } \\
\text { - providing updates of patients' status to PCP } \\
\text { and MHS }\end{array}$ & $\%$ \\
\hline & MHS & not reported & not reported & $\begin{array}{l}\text { - receives weekly updates from DESTRESS } \\
\text { nurses }\end{array}$ & $\%$ \\
\hline \multirow[t]{3}{*}{ CALM } & PCP & $\begin{array}{l}\text { internists; } \\
\text { family physicians }\end{array}$ & $\begin{array}{l}\text { single-session medication } \\
\text { management training using a } \\
\text { simple algorithm }\end{array}$ & $\begin{array}{l}\text { - remains the clinician of record } \\
\text { - prescribed all medications }\end{array}$ & by psychiatrist \\
\hline & ACS (CM) & $\begin{array}{l}\text { social workers; } \\
\text { RN; } \\
\text { psychologists }\end{array}$ & $\begin{array}{l}\text { - formal training applying the } \\
\text { MINI- didactics for CBT } \\
\text { program, MI, medication } \\
\text { algorithm for anxiety }\end{array}$ & $\begin{array}{l}\text { - providing eligibility assessment } \\
\text { - delivery of computerized CBT program } \\
\text { - monitoring of symptoms and adherence }\end{array}$ & $\begin{array}{l}\text { by psychiatrist } \\
\text { and } \\
\text { psychologist }\end{array}$ \\
\hline & MHS & $\begin{array}{l}\text { psychiatrists; } \\
\text { psychologists }\end{array}$ & not reported & $\begin{array}{l}\text { - providing weekly supervision of ACS for } \\
\text { diagnostic, CBT and medication management } \\
\text { issues } \\
\text { - providing medication consultation to PCPS. }\end{array}$ & $\%$ \\
\hline \multirow[t]{2}{*}{ PE-PC } & PCP & not reported & not reported & member of the primary care team & $\%$ \\
\hline & MHS & $\begin{array}{l}\text { doctoral-level } \\
\text { behavioral health } \\
\text { providers (three } \\
\text { civilian, one military } \\
\text { psychologist) }\end{array}$ & $\begin{array}{l}\text { full training workshop for } P E ; \\
\text { one to two training cases in } \\
\text { the PE-PC intervention under } \\
\text { close supervision }\end{array}$ & $\begin{array}{l}\text { - follows up any missed appointments and } \\
\text { attempts to reschedule } \\
\text { - delivering PE-PC }\end{array}$ & $\begin{array}{l}\text { by PI and } \\
\text { independet } \\
\text { clinician }\end{array}$ \\
\hline
\end{tabular}




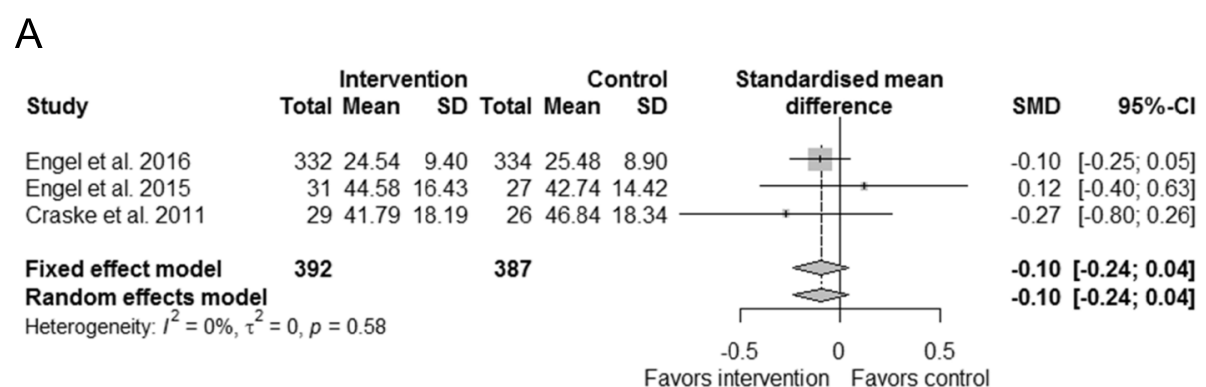

B

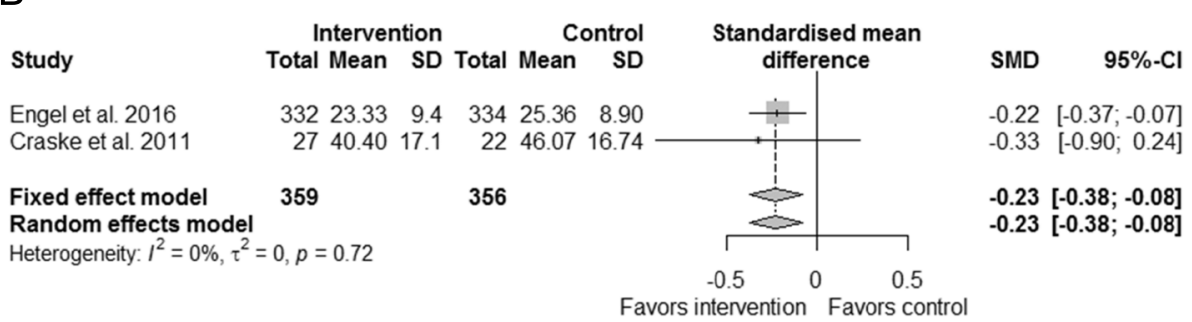

C

\begin{tabular}{|c|c|c|c|c|c|c|}
\hline & 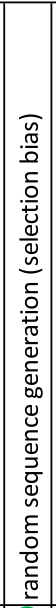 & 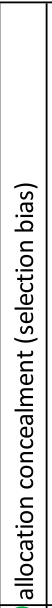 & 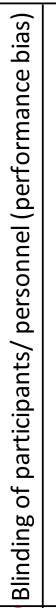 & 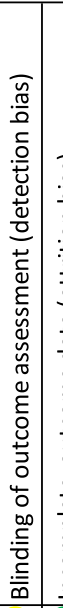 & 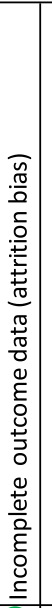 & 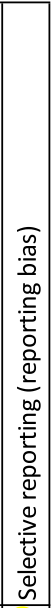 \\
\hline Engel 2016 & + & + & 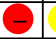 & $?$ & + & $?$ \\
\hline Engel 2015 & + & $?$ & $\theta$ & + & $?$ & $?$ \\
\hline Craske 2011 & + & + & & + & + & $?$ \\
\hline
\end{tabular}

Fig. 1 Meta-analysis of interventions for PTSD including psychological therapies in primary care vs. control; risk of bias summary. Small grey squares represent SMDs for PTSD symptom improvement of individual RCTs, large grey squares represent weights, horizontal lines show $95 \% \mathrm{Cl}$, grey diamonds represent total SMDs of interventions and $95 \% \mathrm{Cl}$. a: short-term effects (0-6 months); b long-term effects ( 12 months and longer); c risk of bias summary according to the Cochrane Collaboration Risk of Bias Tool

According to the pooled analysis of the three studies, multifaceted interventions including psychological therapies and involving PCPs may make little or no difference to PTSD symptoms (SMD, $-0.1 ; 95 \%$ confidence interval, - 0.24-0.04; Fig. 1a). No statistical heterogeneity was detected $\left(\mathrm{I}^{2}=0 \%\right.$; $\left.95 \% \mathrm{CI}, 0.0-80.8 \%\right)$. Only two studies reported on long-term outcomes (12-18 months). According to the meta-analysis of these two studies, psychological interventions in primary care may improve PTSD symptoms over time (SMD, - 0.23; 95\%
CI, $-0.38--0.08 ; \mathrm{I}^{2}=0 \%$; Fig. $\left.1 \mathrm{~b}\right)$. Because only two studies contributed to the pooled analyses for long-term effects, we also investigated time, intervention and timeintervention interaction effects with a generalized linear mixed model. Time (regression coefficient, -.28 ; SE, $0.05 ; P<.0001)$ and time-intervention interaction (regression coefficient, -.28 ; SE, $0.08 ; P=.0020$ ) were significantly associated with means of PTSD symptom measures, with the treatment effect yielding a regression coefficient equal to .94; SE 0.52; $P=.09$. 
We did not perform a meta-analysis for the treatment effects on comorbidities and quality of life due to the limited number of included studies. Further secondary outcomes were mostly not reported separately for the PTSD subgroup or not reported at all.

\section{Risk of bias within studies}

A summary of risk of bias judgments can be found in Fig. 1c. For a more detailed description, with support of judgments, see additional file 4 . All studies had low risk of bias for random sequence generation. Allocation concealment was judged with low risk in two studies, with unclear risk and with high risk each in one study. Blinding of participants and personnel was not possible due to intervention study design. A lack of blinding may influence outcome, thus all studies scored high risk of bias. Blinding of outcome assessment was adequately reported in two studies, but risk was unclear in two studies. Two studies showed low risk of bias for incomplete outcome data. All studies did not report on all pre-specified secondary outcomes or no pre-specified outcomes were available at all and were judged with unclear risk for reporting bias.

\section{Risk of bias across studies}

Publication bias is difficult to judge with only four included studies. We performed a comprehensive search to reduce the risk of small studies not being detected. Two included studies are small in size and report on temporary effects only $[37,40]$.

\section{Certainty assessment of evidence}

Using GRADE we classified the quality of evidence as low because of a limited number of studies, the heterogeneous population of general primary care settings being not represented from the included study population and comparisons between heterogeneous intervention and control groups.

\section{Discussion}

\section{Summary of evidence}

We identified only four studies investigating psychological interventions for posttraumatic stress disorder (PTSD) involving primary care physicians (PCPs). According to our meta- and regression-analysis, multifaceted interventions including psychological therapies in primary care may make little or no difference in the short term, but may improve PTSD symptoms in the long term. These results should be interpreted with caution due to an overall low quality of evidence. All studies were conducted in the United States and three were in military settings. Case managers $(\mathrm{CMs})$ had a central role in three interventions, which were pooled in a meta-analysis. They supported or conducted psychological therapies, coordinated communication between all treatment providers and provided patient activation through patient education, behavioural activation (BA), motivational interviewing (MI), and counselling. PCPs remained responsible for pharmacological treatment, received special training, and got regular feedback on ongoing therapy within the CM-based studies. One study did not involve CMs. The therapy (Prolonged Exposure) was delivered by specially trained behavioural health consultants (BHCs) who were part of the primary care team and worked as a consultant to the PCP.

There are two explanations for the small treatment effect on PTSD symptoms which was detected in the long term (after 12-18 months) but not directly after the intervention. One reason could be the lack of long term outcomes from DESTRESS-PC, the only study with a negative effect in the short term. Another explanation may be, that the long term effect after 12 months is a "learning effect": While well-established psychological interventions are often short and intensive resulting in an immediate and strong effect, in primary care less intensive interventions are common with a lasting "learning effect", which was also shown for collaborative care for depression [23, 41].

Though our aim was to determine the effectiveness of psychological therapies for PTSD in primary care, we only found studies investigating interventions which embedded psychological therapies into multifaceted service delivery models. Besides the applied CBT (cognitive behavioural therapy)-based psychological therapies also $\mathrm{CM}$ initialised patient activation (BA, MI, problem solving) and evidence based pharmacotherapy with adherence monitoring may have contributed to the detected positive treatment effect. Control groups were heterogeneous, too. The control group in STEPS-UP received collaborative care, which was previously implemented to improve PTSD primary care treatment within the military health care system. In DESTRESS-PC PCP training was also part of the optimized usual care. Interestingly, control groups of all three trials show a slight improvement of PTSD symptoms which may be caused by PCP training (DESTRESS-PC), CM alone (STEPS-UP) and mental health specialist referral (CALM). PCP training might be an important component because the ViStA trial (not included in our synthesis, comparing collaborative care for PTSD with minimally enhanced usual care) found that the intervention group (with $\mathrm{CM}$ and $\mathrm{PCP}$ training) and the control group (with PCP training) improved equally [42]. In depression care, training of PCPs resulted in no or only minimal improvement $[24,43]$, while feedback to PCPs about the ongoing therapy was associated with positive outcome measures [24].

The low effect size may partly be explained by the above discussed improvement of PTSD symptoms in the 
active control groups. In addition, the study population of the two largest trials (STEPS-UP and DESTRESS) consisted of military personnel, mainly men in their 20s, who are difficult to engage in mental health care [36, 37]. Finally the STEPS-UP population suffered from a variety of medical and psychiatric comorbidities reducing potential for improvement.

Our meta-analysis supports the explorative conclusion of a narrative systematic review suggesting collaborative care offering psychotherapy as a promising approach [25]. Looking at related diseases, short CBT interventions were effective for anxiety treatment in primary care settings, but results are heterogeneous for the effectiveness of psychotherapies in treating depression in primary care $[21,23,24,44]$. Successful treatment of PTSD in primary care might be even more difficult than depression care: PTSD-symptom improvement was delayed compared to depressive symptoms [36] similar to previous studies showing that depressive patients with comorbid PTSD have a delayed positive response to collaborative care $[45,46]$. An explanation for these findings could be that PTSD is often complicated by comorbidities, which need to be considered when designing new trials and interventions [47].

Another system of service delivery was investigated by the fourth included study [40]: the Primary Care Behavioral Health Model, with behavioural health providers integrated in primary care. In contrast to the other studies no $\mathrm{CM}$ and strategies to improve patient activation were applied; so it was not included within the metaanalysis. Despite the small size of the studied population $(n=67)$ the moderate to large effect sizes obtained up to six months in the follow up assessments encourage the idea that evidence based therapies for PTSD can effectively be transferred from secondary to primary care, especially for patients with mild to moderate symptom severity and enable an early starting therapy.

Because most mental health disorders are treated in general medical settings, primary care remains the first contact to establish effective therapies [48, 49]. In contrast to the central role of PCPs in many countries, in all four studies psychological therapies were provided with the assistance of a CM or by behavioural health providers, but were never delivered by the PCP in person. Only one pilot trial investigated the delivery of brief primary-care CBT delivered by a PCP, unfortunately without follow-up [50].

\section{Limitations}

Our study has several limitations. The quality of evidence is low. There are only three studies contributing to the meta-analysis, with two studies including only a small number of patients with PTSD and one study with a short follow-up (4.5 months) limiting the generalizability of our findings. Performance bias was high in all studies. Due to study design it is difficult to blind participants and personnel for the intervention, thus introducing possible bias in subjective self-report measures. Assessing the certainty of evidence using GRADE [35] we detected serious indirectness because of applicability and indirect comparisons. The four included trials do not represent the heterogeneous population of general primary care settings. Three of four studies were conducted in military settings with mostly male patients who had experienced warrelated trauma, which can be long-lasting, repetitive and may lead to complex PTSD. For this condition a sequenced or phase based multimodal therapy is recommended which can hardly be realised in primary care [51]. In nonspecialised primary care settings the majority of PTSD patients may consist of female survivors of sexual assault [52].

Further indirectness arose from the multifaceted interventions and the heterogeneity in control groups as discussed above.

Reporting of results was often incomplete. In STEPS-UP a separate analysis of primary and secondary outcomes for patients with PTSD only (without depression) was missing. Study selection was difficult because descriptions of PCP involvement was insufficient and required us to contact several authors to request more detailed descriptions. Answers were not always available, so the risk remains that possibly eligible studies were not included.

Although statistical heterogeneity was not detected, all studies included in the meta-analysis show clinical diversity due to different settings (military vs. "general" primary care), different control groups (usual care vs. collaborative care vs. care management) and complex interventions including different components. Nevertheless, all studies applied CBT-based interventions with the help of a CM within a primary care setting and, therefore, it seemed appropriate to combine these studies.

The effectiveness of single intervention components could not be investigated with meta-regression due the lack of studies investigating only single components of the different service delivery models. The role of PCP involvement, especially, could not be assessed. The influence of interventions on comorbidities and quality of life could also not be calculated due to the limited number of included studies.

Our findings cannot be generalized to primary care settings in other countries because all studies were conducted in the United States and three studies were conducted in military contexts. In addition, the majority applied CM which is not always well established in other countries.

\section{Conclusion}

To our knowledge this is the first systematic review and meta-analysis of primary care based psychological interventions for PTSD. 
Four randomized controlled trails (RCTs) could be included which applied multifaceted interventions based on psychological therapies for PTSD involving PCPs. Psychological interventions for PTSD in primary care settings may be effective in the long term. Evidence supports the feasibility of primary care interventions for PTSD in general and the need for more studies examining psychological interventions for PTSD in primary care. The limited amount of research, an overall low quality of evidence and the rising number of different service delivery models in primary care require a differentiated analysis and hinder a universally valid recommendation for future treatment implications.

Some trials have only been published to date as study designs or pilot trials $[53,54]$, but may soon add relevant findings.

Future studies should investigate the contribution to effectiveness made by both intervention components and involved providers.

\section{Supplementary information}

Supplementary information accompanies this paper at https://doi.org/10. 1186/s12875-020-01244-4.

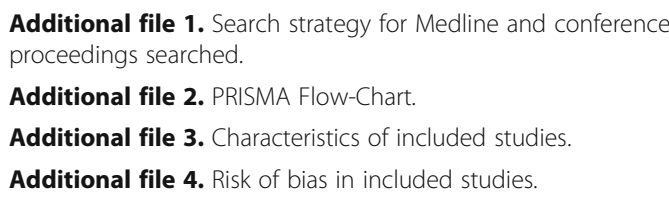

\section{Abbreviations}

BA: Behavioural activation; BHC: Behavioural health consultant; BIC: Bayesian information criterion; CAPS: Clinician administered PTSD scale; CBT: Cognitive behavioural therapies; CCM: Chronic care model; CM: Case manager; DSM5: Diagnostic and Statistical Manual of Mental Disorders 5th edition; EMDR: Eye movement desensitization and reprocessing; ICD: International Statistical Classification of Diseases and Related Health Problems; Ml: Motivational interviewing; NET: Narrative exposure therapy; PCL-C: PTSD Checklist-Civilian version; PCL-S: PTSD Checklist-Stressor Specific version; PCP: Primary care physician; PTSD: Posttraumatic stress disorder; RCT: Randomized controlled trial

\section{Acknowledgements}

We are grateful for the technical and material support of Nico Schneider and Michelle Kaufmann and the competent support searching Embase of Heike Goebel. We also thank Nancy Myers for her language support. Furthermore, we would like to thank the authorship team of the helpful, ESRC-funded guidance on the conduct of narrative synthesis: Jennie Popay, Helen Roberts, Amanda Sowden, Mark Petticrew, Lisa Arai, Mark Rodgers, Nicky Britten, Katrina Roen and Steven Duffy.

\section{Authors' contributions}

RG and AF had full access to all the data in the study and take responsibility for the integrity of the data and the accuracy of the data analysis. Study concept and design: MB, AF, RG, JG1, PS, KS, SS, HCV. Acquisition, analysis, or interpretation of data: All authors (RG, $A F, M K, P S, K S, S S, S J Z, F W, J G, M B$, $H C V, J G 1)$. Drafting of the manuscript: AF, RG, JG1, MK, KS, SS, HCV. Critical revision of the manuscript for important intellectual content: All authors (RG, AF, MK, PS, KS, SS, SJZ, FW, JG, MB, HCV, JG1). Study supervision: JG1, HCV. All authors read and approved the final manuscript.
Funding

Open Access funding provided by Projekt DEAL.

\section{Availability of data and materials}

The datasets used and analysed during the current study are available from the corresponding author on reasonable request.

\section{Ethics approval and consent to participate}

This contribution is a pure literature review that does not involve tests/ studies on humans or animals.

\section{Consent for publication}

Not applicable.

\section{Competing interests}

The authors declare that they have no competing interests.

\section{Author details}

${ }^{1}$ Institute of General Practice and Family Medicine, Jena University Hospital, Friedrich-Schiller-University, Bachstr. 18, 07743 Jena, Germany. ${ }^{2}$ Institute of Medical Statistics, Computer Sciences and Documentation, Jena University Hospital, Friedrich-Schiller-University, Jena, Germany. ${ }^{3}$ Institute of General Practice/Family Medicine, University Hospital of LMU Munich, Munich, Germany. ${ }^{4}$ Department of Psychiatry, Faculty of Medicine, University of Freiburg, Freiburg, Germany. ${ }^{5}$ Department of Family Medicine,

Ruhr-University of Bochum, Bochum, Germany.

Received: 12 February 2020 Accepted: 11 August 2020

Published online: 26 August 2020

\section{References}

1. Stein DJ, Seedat S, Iversen A, Wessely S. Post-traumatic stress disorder: medicine and politics. Lancet (London, England). 2007;369(9556):139-44.

2. American Psychiatric Association. Diagnostic and Statistical Manual of Mental Disorders. 5th ed. Arlington, VA; 2013.

3. World Health Organization. ICD-11 for Mortality and Morbidity Statistics. 2018. https://icd.who.int/browse11/I-m/en. Accessed July 2018.

4. Atwoli L, Stein DJ, Koenen KC, McLaughlin KA. Epidemiology of posttraumatic stress disorder: prevalence, correlates and consequences. Curr Opin Psychiatry. 2015;28(4):307-11.

5. Kessler RC, Rose S, Koenen KC, Karam EG, Stang PE, Stein DJ, et al. How well can post-traumatic stress disorder be predicted from pre-trauma risk factors? An exploratory study in the WHO world mental health surveys. World Psychiatry. 2014;13(3):265-74

6. Cusack K, Jonas DE, Forneris CA, Wines C, Sonis J, Middleton JC, et al. Psychological treatments for adults with posttraumatic stress disorder: a systematic review and meta-analysis. Clin Psychol Rev. 2016;43:128-41.

7. Bisson Jl, Roberts NP, Andrew M, Cooper R, Lewis C. Psychological therapies for chronic post-traumatic stress disorder (PTSD) in adults. Cochrane database Syst Rev. 2013;12:CD003388.

8. Lee DJ, Schnitzlein CW, Wolf JP, Vythilingam M, Rasmusson AM, Hoge CW. PSYCHOTHERAPY VERSUS PHARMACOTHERAPY FOR POSTTRAUMATIC STRE SS DISORDER: Systemic review and META-analyses to determine FIRST-line treatments. Depression Anxiety. 2016;33(9):792-806.

9. Wang PS, Lane M, Olfson M, Pincus HA, Wells KB, Kessler RC. Twelve-month use of mental health services in the United States: results from the National Comorbidity Survey Replication. Arch Gen Psychiatry. 2005;62(6):629-40.

10. Spottswood M, Davydow DS, Huang $H$. The prevalence of posttraumatic stress disorder in primary care: a systematic review. Harvard Rev Psychiatry. 2017;25(4):159-69.

11. Wang PS, Berglund P, Olfson M, Pincus HA, Wells KB, Kessler RC. Failure and delay in initial treatment contact after first onset of mental disorders in the National Comorbidity Survey Replication. Arch Gen Psychiatry. 2005;62(6):603-13.

12. Tanielian T, Woldetsadik MA, Jaycox LH, Batka C, Moen S, Farmer C, et al. Barriers to engaging service members in mental health care within the U.S. military health system. Psychiatr Serv. 2016;67(7):718-27.

13. Sonis J. PTSD in primary care - an update on evidence-based management. Curr Psychiatry Rep. 2013;15(7):373.

14. Possemato K. The current state of intervention research for posttraumatic stress disorder within the primary care setting. J Clin Psychol Med Settings. 2011;18(3):268-80 
15. National Institute for Health and Clinical Excellence. Post-traumatic stress disorder: management Clinical guideline. Manchester 2005. https://www. nice.org.uk/guidance/cg26/resources/posttraumatic-stress-disordermanagement-pdf-975329451205. Accessed May 2017.

16. Department of Veterans Affairs and Department of Defense. VA/DoD Clinical Practice Guideline for the Management of Post-Traumatic Stress. 2017. https://www.healthquality.va.gov/guidelines/MH/ptsd/ VADoDPTSDCPGFinal012418.pdf. Accessed May 2017.

17. Mainous AG 3rd, Baker R, Love MM, Gray DP, Gill JM. Continuity of care and trust in one's physician: evidence from primary care in the United States and the United Kingdom. Fam Med. 2001;33(1):22-7.

18. Bodenheimer T, Ghorob A, Willard-Grace R, Grumbach K. The 10 building blocks of high-performing primary care. Ann Fam Med. 2014;12(2):166-71.

19. Starfield B. Primary care: concept, evaluation, and policy: Oxford University press; 1992.

20. Saxena S, Thornicroft G, Knapp M, Whiteford H. Resources for mental health: scarcity, inequity, and inefficiency. Lancet (London, England). 2007; 370(9590):878-89.

21. Twomey C, O'Reilly G, Byrne M. Effectiveness of cognitive behavioural therapy for anxiety and depression in primary care: a meta-analysis. Fam Pract. 2015;32(1):3-15.

22. Bower P, Gilbody S, Richards D, Fletcher J, Sutton A. Collaborative care for depression in primary care. Making sense of a complex intervention: systematic review and meta-regression. Br J Psychiatry. 2006;189:484-93.

23. Gilbody S, Bower P, Fletcher J, Richards D, Sutton AJ. Collaborative care for depression: a cumulative meta-analysis and review of longer-term outcomes. Arch Intern Med. 2006;166(21):2314-21.

24. Christensen H, Griffiths KM, Gulliver A, Clack D, Kljakovic M, Wells L. Models in the delivery of depression care: a systematic review of randomised and controlled intervention trials. BMC Fam Pract. 2008;9:25.

25. Hoeft TJ, Stephens KA, Vannoy SD, Unutzer J, Kaysen D. Interventions to treat posttraumatic stress disorder in partnership with primary care: a review of feasibility and large randomized controlled studies. Gen Hosp Psychiatry. 2019;60:65-75

26. van Emmerik AA, Reijntjes A, Kamphuis $\mathrm{JH}$. Writing therapy for posttraumatic stress: a meta-analysis. Psychother Psychosom. 2013;82(2):82-8.

27. Brom D, Kleber RJ, Defares PB. Brief psychotherapy for posttraumatic stress disorders. J Consult Clin Psychol. 1989;57(5):607-12.

28. Cloitre M, Stovall-McClough KC, Nooner K, Zorbas P, Cherry S, Jackson CL, et al. Treatment for PTSD related to childhood abuse: a randomized controlled trial. Am J Psychiatry. 2010;167(8):915-24.

29. Tran US, Gregor B. The relative efficacy of bona fide psychotherapies for post-traumatic stress disorder: a meta-analytical evaluation of randomized controlled trials. BMC Psychiatry. 2016;16:266.

30. Lampe A, Mitmansgruber H, Gast U, Schussler G, Reddemann L. Treatment outcome of psychodynamic trauma therapy in an inpatient setting. Neuropsychiatr. 2008;22(3):189-97.

31. Effective Practice and Organisation of Care (EPOC). Data collection form. EPOC Resources for review authors. Oslo: Norwegian Knowledge Centre for the Health Services; 2013. http://epoc.cochrane.org/epoc-specific-resourcesreview-authors. Accessed Nov 2016.

32. Hoffmann TC, Glasziou PP, Boutron I, Milne R, Perera R, Moher D, et al. Better reporting of interventions: template for intervention description and replication (TIDieR) checklist and guide. BMJ. 2014;348:g1687.

33. Wagner EH, Austin BT, Davis $C$, Hindmarsh M, Schaefer J, Bonomi A. Improving chronic illness care: translating evidence into action. Health Aff (Millwood). 2001;20(6):64-78.

34. Higgins JPT, Altman DG, Sterne JAC. Chapter 8: Assessing risk of bias in included studies. In: Higgins JPT, Green S, editors. Cochrane Handbook for Systematic Reviews of Interventions Version 5.10 (updated March 2011). The Cochrane Collaboration; 2011.

35. Schünemann HJOA, Vist GE, Higgins JPT, Deeks JJ, Glasziou P. Interpreting results and drawing conclusions. In: GS HJPT, editor. Cochrane Handbook for Systematic Reviews of Interventions Version 510 [updated March 2011] The Cochrane Collaboration; 2011.

36. Engel CC, Jaycox LH, Freed MC, Bray RM, Brambilla D, Zatzick D, et al. Centrally assisted collaborative Telecare for posttraumatic stress disorder and depression among military personnel attending primary care: a randomized clinical trial. JAMA Intern Med. 2016;176(7):948-56.

37. Engel CC, Litz B, Magruder KM, Harper E, Gore K, Stein N, et al. Delivery of self training and education for stressful situations (DESTRESS-PC): a randomized trial of nurse assisted online self-management for PTSD in primary care. Gen Hosp Psychiatry. 2015;37(4):323-8.

38. Craske MG, Stein MB, Sullivan G, Sherbourne C, Bystritsky A, Rose RD, et al. Disorder-specific impact of coordinated anxiety learning and management treatment for anxiety disorders in primary care. Arch Gen Psychiatry. 2011; 68(4):378-88

39. Roy-Byrne P, Craske MG, Sullivan G, Rose RD, Edlund MJ, Lang AJ, et al. Delivery of evidence-based treatment for multiple anxiety disorders in primary care: a randomized controlled trial. JAMA. 2010;303(19):1921-8.

40. Cigrang JA, Rauch SA, Mintz J, Brundige AR, Mitchell JA, Najera E, et al. Moving effective treatment for posttraumatic stress disorder to primary care: a randomized controlled trial with active duty military. Families Systems Health. 2017;35(4):450-62.

41. Archer J, Bower P, Gilbody S, Lovell K, Richards D, Gask L, et al. Collaborative care for depression and anxiety problems. Cochrane database Syst Rev. 2012;10:Cd006525.

42. Meredith LS, Eisenman DP, Han B, Green BL, Kaltman S, Wong EC, et al. Impact of collaborative Care for Underserved Patients with PTSD in primary care: a randomized controlled trial. J Gen Intern Med. 2016; 31(5):509-17.

43. Gilbody S, Whitty P, Grimshaw J, Thomas R. Educational and organizational interventions to improve the management of depression in primary care: a systematic review. JAMA. 2003;289(23):3145-51.

44. Cape J, Whittington C, Buszewicz M, Wallace P, Underwood L. Brief psychological therapies for anxiety and depression in primary care: metaanalysis and meta-regression. BMC Med. 2010;8:38.

45. Hegel MT, Unutzer J, Tang L, Arean PA, Katon W, Noel PH, et al. Impact of comorbid panic and posttraumatic stress disorder on outcomes of collaborative care for late-life depression in primary care. Am J Geriatr Psychiatry. 2005;13(1):48-58.

46. Chan D, Fan MY, Unutzer J. Long-term effectiveness of collaborative depression care in older primary care patients with and without PTSD symptoms. Int J Geriatric Psychiatry. 2011;26(7):758-64.

47. Kessler RC, Chiu WT, Demler O, Merikangas KR, Walters EE. Prevalence, severity, and comorbidity of 12-month DSM-IV disorders in the National Comorbidity Survey Replication. Arch Gen Psychiatry. 2005;62(6):617-27.

48. Kessler RC, Demler O, Frank RG, Olfson M, Pincus HA, Walters EE, et al. Prevalence and treatment of mental disorders, 1990 to 2003. N Engl J Med. 2005;352(24):2515-23.

49. Norquist GS, Regier DA. The epidemiology of psychiatric disorders and the de facto mental health care system. Annu Rev Med. 1996;47:473-9.

50. Prins A, Cimpean D, Schnurr PP. Treatment in primary care settings. In: Mueser KT, Rosenberg SD, Rosenberg HJ, editors. Treatment of posttraumatic stress disorder in special populations: a cognitive restructuring program. Washington DC: American Psychological Association; 2009. p. 301-14.

51. Cloitre M, Courtois CA, Charuvastra A, Carapezza R, Stolbach BC, Green BL. Treatment of complex PTSD: results of the ISTSS expert clinician survey on best practices. J Trauma Stress. 2011;24(6):615-27.

52. Kessler RC, Sonnega A, Bromet E, Hughes M, Nelson CB. Posttraumatic stress disorder in the National Comorbidity Survey. Arch Gen Psychiatry. 1995; 52(12):1048-60.

53. Hoerster KD, Jakupcak M, Stephenson KR, Fickel JJ, Simons CE, Hedeen A, et al. A pilot trial of telephone-based collaborative care management for PTSD among Iraq/Afghanistan war veterans. Telemed J E Health. 2015;21(1): 42-7.

54. Kaltman S, Pauk J, Alter CL. Meeting the mental health needs of lowincome immigrants in primary care: a community adaptation of an evidence-based model. Am J Orthop. 2011;81(4):543-51.

\section{Publisher's Note}

Springer Nature remains neutral with regard to jurisdictional claims in published maps and institutional affiliations. 